\begin{tabular}{|c|c|}
\hline Title & Ostwald ripening under temperature gradient: A phase field study \\
\hline Author(s) & Y amada, Ryo; Inubushi, Haruki; Ohno, Munekazu \\
\hline Citation & $\begin{array}{l}\text { Journal of A pplied Physics, 130(1), } 015109 \\
\text { https://doi.org/10.1063/5.0055198 }\end{array}$ \\
\hline Issue Date & 2021-07-07 \\
\hline Doc URL & http:/hdl.handle.net/2115/82586 \\
\hline Rights & $\begin{array}{l}\text { This article may be downloaded for personal use only. A ny other use requires prior permission of the author and AIP } \\
\text { Publishing. This article appeared in (citation of published article) and may be found at (URL/ink for published article } \\
\text { abstract) }\end{array}$ \\
\hline Type & article \\
\hline File Information & 5.0055198.pdf \\
\hline
\end{tabular}

Instructions for use 


\title{
Ostwald ripening under temperature gradient: A phase-field study
}

\author{
Cite as: J. Appl. Phys. 130, 015109 (2021); doi: 10.1063/5.0055198 \\ Submitted: 26 April 2021 . Accepted: 12 June 2021 . \\ Published Online: 6 July 2021

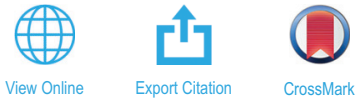 \\ Ryo Yamada, $^{1, a)}$ (D) Haruki Inubushi, ${ }^{2}$ and Munekazu Ohno ${ }^{1}$ (D)

\begin{abstract}
AFFILIATIONS
${ }^{1}$ Division of Materials Science and Engineering, Faculty of Engineering, Hokkaido University, Kita 13 Nishi 8, Kita-ku, Sapporo, Hokkaido 060-8628, Japan

${ }^{2}$ Graduate School of Engineering, Hokkaido University, Kita 13 Nishi 8, Kita-ku, Sapporo, Hokkaido 060-8628, Japan
\end{abstract}

a) Author to whom correspondence should be addressed: ryamada@eng.hokudai.ac.jp

\begin{abstract}
Ostwald ripening under a temperature gradient in binary model alloys is investigated using a quantitative phase-field model. The simulations show that a cube of average radius of a second-phase particle is proportional to time, and the particle size distribution shows self-similarity in a steady state, as with a uniform temperature field. It is found that the growth rate of particles under a temperature gradient is faster than that in the isothermal case, and the steady-state particle size distribution depends on the magnitude of the temperature gradient. Furthermore, the second-phase particles migrate from low temperature regions to high temperature regions when a non-uniform temperature field is applied. The migration velocity of particles, averaged over the whole system, increases with the magnitude of the temperature gradient. On the other hand, the velocity of each particle is not relevant to particle size. Hence, the particle migration is entirely ascribed to the diffusion flux driven by the concentration gradient originating from the temperature dependence of solute solubility.
\end{abstract}

Published under an exclusive license by AIP Publishing. https://doi.org/10.1063/5.0055198

\section{INTRODUCTION}

Ostwald ripening is a well-known phenomenon in materials science, where large particles of the second phase in a matrix grow at the expense of small particles. This is the last stage of phase separation processes in alloys. Ostwald ripening can be explained well by considering an effect of interfacial energy of the second phase on the local equilibrium at the interface, where local equilibrium compositions at the particle-matrix interface are altered (the Gibbs-Thomson effect). ${ }^{1}$ Due to the Gibbs-Thomson effect, a mass transport takes place between second-phase particles in the matrix, which leads to a concentration gradient between small and large particles and results in the diminishing (coarsening) of small (large) particles.

During the coarsening process of the second phase, the average particle size increases while the total number of particles decreases. Because the distribution of the second phase strongly affects the mechanical properties of materials, ${ }^{2,3}$ tremendous efforts have been made to understand the mechanism, with both theoretical and experimental approaches being employed. ${ }^{4-14}$ It has been well demonstrated that the particle size distribution normalized by the average value shows a time-invariant feature in the steady-state condition, i.e., a self-similarity, and that the cube of the average particle radius $r^{3}$ is proportional to time $t$. To be more specific, it follows the relation $r^{3}-r_{c}^{3}=K\left(t-t_{c}\right)$, where $K$ is the growth-rate constant and $r_{c}$ and $t_{c}$ are, respectively, the average particle radius and time at the onset of Ostwald ripening.

Note that most previous studies of Ostwald ripening have focused on a uniform temperature field. In many practical situations, however, the temperature is not always uniform in materials. Hence, in practice, the growth and coarsening of second-phase particles often take place under a temperature gradient. It has been shown that second-phase particles migrate in the matrix in the direction of a temperature gradient. ${ }^{15}$ Several works have been conducted to clarify the migration behavior of particles ${ }^{15-18}$ and various mechanisms for particle migration have been proposed. Further research is needed to reveal the dominant mechanism of particle migration. Moreover, experimental work on a fibrous $\mathrm{Al}-\mathrm{Al}_{3} \mathrm{Ni}$ system showed that the coarsening of the second phase is accelerated by the temperature gradient. ${ }^{15}$ This point was investigated in further detail by Snyder et al. using numerical simulations based on a steady-state diffusion equation. ${ }^{19}$ They showed that the growth rate of the second phase in a non-uniform temperature 
field is faster than one in a uniform temperature field. Although they noted particle migration in their numerical results, it was ignored in their analysis because the velocity was negligibly small. Important work remains to be carried out to reveal details of Ostwald ripening in a temperature gradient, such as a relation between particle migration and the acceleration of the coarsening process, and the dependence of particle size distribution on the temperature gradient using an advanced numerical simulation technique that should be free from the assumptions and approximations made in the early theoretical and numerical works.

In this article, therefore, the effects of a temperature gradient on the coarsening behavior of a second phase distributed in a matrix are studied in detail using a quantitative phase-field model (QPFM) ${ }^{20-23}$ which is one of the most effective and reliable methods of describing microstructural evolution processes in alloy systems. This article is organized as follows. First, the theoretical background of QPFM is briefly described in Sec. II, as well as computational conditions. In Sec. III, the calculated coarsening behaviors of particles randomly distributed in a matrix in both uniform and non-uniform temperature fields are presented, and the effects of temperature gradients on the coarsening behavior are discussed. The results and new findings obtained in the present work are summarized in Sec. IV.

\section{METHOD}

\section{A. Quantitative phase-field model}

A phase-field model (PFM) is a viable computational method of describing a microstructure evolution process, where multiphysics are generally involved, such as multicomponent solute diffusion, heat diffusion, and fluid dynamics. A unique feature of PFM is that an interface between neighboring phases (or domains) is described by a continuous variation of phase-field variable which takes constant values assigned to each phase. This diffuse interface enables one to simulate complex microstructures without tracking moving interfaces. However, it is known that simulated results obtained from a conventional PFM depend on the interface thickness. ${ }^{20-23}$ To avoid this serious problem, a QPFM has been developed, where the solution to the free-boundary problem is recovered in the thin-interface limit. Several QPFMs have been suggested, ${ }^{20-22}$ depending on the system of interest and accordingly the phenomenological correction term. The QPFM developed by Ohno and Matsuura ${ }^{22}$ is used in this work.

In the QPFM, the phase-field variable, $\phi$, takes +1 in the solid and -1 in the liquid and varies from +1 to -1 inside the solidliquid interface. The time evolution equation of $\phi$ in a nontemperature field is given by ${ }^{22,24}$

$$
\begin{aligned}
\tau\left[1-(1-k) \frac{x-x_{0}}{l_{T}}\right] \frac{\partial \phi}{\partial t}= & W^{2} \nabla^{2} \phi+\phi\left(1-\phi^{2}\right) \\
& -\lambda\left(1-\phi^{2}\right)^{2}\left(u+\frac{x-x_{0}}{l_{T}}\right),
\end{aligned}
$$

where $t$ is the time; $x$ is the distance along the temperature gradient; $x_{0}$ is a reference coordinate of $x$ and is the center of the system in the $x$-direction; $k$ is the equilibrium partition coefficient; $W$ is the interface thickness; $\tau$ is the phase-field relaxation time defined as $\tau=a_{2} \lambda W^{2} / D_{L}$, where $a_{2}=0.6267$ and $D_{L}$ is the solute diffusivity in the liquid phase; $\lambda$ is a coupling constant given as $\lambda=a_{1} W / d_{0}$, where $a_{1}=0.8839$ and $d_{0}$ is the capillary length; and $l_{T}$ is a thermal length defined as $l_{T}=\left(T_{L}-T_{S}\right) / G$ using a temperature gradient $G$ with the liquidus and solidus temperatures, $T_{L}$ and $T_{S}$, for a given alloy composition. Furthermore, $u$ is the dimensionless local supersaturation defined as $u \equiv\left(c_{L}-c_{L}^{e}\right) /\left(c_{L}^{e}-c_{S}^{e}\right)$, where $c_{L}$ and $c_{S}$ are the liquid and solid concentrations, respectively, and $c_{L}^{e}$ and $c_{S}^{e}$ are their equilibrium values.

When a system is isothermal, Ostwald ripening is entirely ascribed to the diffusion flux due to the concentration gradient originating from the Gibbs-Thomson effect. Additional diffusion flux may occur when a system is not isothermal. The diffusion flux is caused by the Soret effect, i.e., the direct coupling between solute diffusion and heat diffusion. Moreover, the additional diffusion flux emerges according to the dependence of the solubility of the solute atom on temperature, which causes the difference in local equilibrium concentration at the interface at different temperatures and, thereby, the diffusion flux in the matrix. In this study, we shall focus on the latter effect and neglect the Soret effect for the sake of simplicity. The time evolution of $u$ is then given by

$$
\frac{1+k-(1-k) \phi}{2} \frac{\partial u}{\partial t}=\nabla \cdot\left[D_{L} q(\phi) \nabla u+\frac{1}{2 \sqrt{2}}\left(1-k \frac{D_{S}}{D_{L}}\right) W\{1+(1-k) u\} \frac{\partial \phi}{\partial t} \frac{\nabla \phi}{|\nabla \phi|}\right]+\frac{1}{2}[1+(1-k) u] \frac{\partial \phi}{\partial t},
$$

where $D_{S}$ is the solute diffusivity in the solid phase and $q(\phi)$ is defined as

$$
q(\phi)=\frac{1}{2}\left[(1+\phi) k \frac{D_{S}}{D_{L}}-(1-\phi)\right] .
$$

To effectively solve Eqs. (2) and (3) simultaneously, the preconditioning $^{25}$ is employed in this work. By changing the variable from $\phi$ to $\psi\left[\equiv \sqrt{2} \tanh ^{-1}(\phi)\right]$, Eqs. (2) and (3) become

$$
\begin{aligned}
\alpha \eta^{2}[1+(1-k) u] \frac{\partial \psi}{\partial t}= & \eta^{2}\left(\nabla^{2} \psi-\sqrt{2} \phi|\nabla \psi|^{2}\right)+\sqrt{2} \phi \\
& -\sqrt{2} a_{1} W\left(1-\phi^{2}\right)\left(u+\frac{x}{l_{T}}\right)
\end{aligned}
$$




$$
\begin{aligned}
\frac{1+k-(1-k) \phi}{2} \frac{\partial u}{\partial t}= & \nabla \cdot[q(\phi) \nabla u]+\nabla \cdot\left[\frac{\eta}{4}\left(1-k \frac{D_{S}}{D_{L}}\right)\{1+(1-k) u\}\left(1-\phi^{2}\right) \frac{\partial \psi}{\partial t} \frac{\nabla \psi}{|\nabla \psi|}\right] \\
& +\frac{1}{2 \sqrt{2}}[1+(1-k) u]\left(1-\phi^{2}\right) \frac{\partial \psi}{\partial t},
\end{aligned}
$$

where $x$ and $t$ are normalized as $x / d_{0}$ and $D_{L} t / d_{0}^{2}$, respectively, and $\alpha$ and $\eta$ are defined as $\alpha \equiv \tau D_{L} / W^{2}$ and $\eta \equiv W / d_{0}$. The coupling constant $\lambda$ is given by

$$
\lambda=a_{1} \frac{W}{d_{0}}=a_{1} W \frac{R T_{m}(1-k)\left(c_{L}^{e}-c_{S}^{e}\right)}{v_{m} \gamma},
$$

where $R$ is the gas constant, $T_{m}$ is the melting temperature of pure solvent, $v_{m}$ is the molar volume, and $\gamma$ is the interfacial energy of the solid-liquid interface.

Note that the QPFM employed here assumes a dilute solution limit for a free energy and includes a "phenomenological" correction term introduced for coupling between phase-field and conserved variables. ${ }^{22}$ One of the present authors has recently proposed a variational formulation of a quantitative phase-field model in which the cross-coupling terms are derived in a natural way. ${ }^{23}$ Also, this variational QPFM is free from the assumption of diffusion fields and can be applied to a variety of phenomena. However, it is known that a fast convergence of the results can be obtained by decreasing the interface thickness, even in the earliest developed QPFMs. ${ }^{22,26,27}$ In the present work, therefore, the QPFM suggested by Ohno and Matsuura $^{22}$ is employed, in consideration of the balance between computational cost and numerical accuracy.

\section{B. Computational conditions}

In this work, a coarsening behavior of second-phase particles distributed randomly in a matrix is simulated, focusing on binary alloy systems. The physical parameters used in the calculations are summarized in Table I. Although the focus of this study is placed on model alloys, the values of these parameters are the same as those in the solidification of the Al-rich fcc phase in an $\mathrm{Al}-\mathrm{Cu}$ alloy. Because there is a rather small elastic strain during coarsening in an $\mathrm{Al}-\mathrm{Cu}$ alloy system, experimental works have been conducted to elucidate Ostwald ripening in this alloy system. ${ }^{13}$ In

TABLE I. Physical parameters used in the present study. ${ }^{32}$

\begin{tabular}{cc}
\hline \hline Physical parameter & Value \\
\hline Molar volume, $v_{m}\left(\mathrm{~m}^{3} / \mathrm{mol}\right)$ & $1.0 \times 10^{-5}$ \\
Interfacial energy of solid-liquid & 0.16 \\
interface, $\gamma\left(\mathrm{J} / \mathrm{m}^{3}\right)$ & \\
Liquidus slope, $m_{L}(\mathrm{~K} / \mathrm{mol} . \%)$ & -620.0 \\
Melting temperature of pure $\mathrm{Al}, T_{m}(\mathrm{~K})$ & 933.25 \\
Liquid diffusivity, $D_{L}\left(\mathrm{~m}^{2} / \mathrm{s}\right)$ & $1.06 \times 10^{-7} \exp$ \\
& $\left(-2.41 \times 10^{4} / R T\right)$ \\
Solid diffusivity, $D_{S}\left(\mathrm{~m}^{2} / \mathrm{s}\right)$ & $4.44 \times 10^{-5} \exp$ \\
& $\left(-13.4 \times 10^{5} / R T\right)$ \\
\hline
\end{tabular}

addition, solute diffusion in the liquid phase takes place relatively quickly in this alloy system, so it is expected that a coarsening behavior of the second phase can be studied in a short computational time. Note that different values of the equilibrium partition coefficient, $k\left(\equiv c_{S}^{e} / c_{L}^{e}\right)=0.14,0.24$, and 0.34 , are considered in this study in order to investigate its effect on coarsening behavior. Hence, the focus of this study is basically model alloys.

Equations (4) and (5) are discretized using a second-order finite difference scheme and are solved using a first-order Euler scheme. Two-dimensional (2D) simulations are performed in a computational domain of $6144 \times 12288 \mu \mathrm{m}^{2}$ with grid spacing $d x=d y=1.5 \times 10^{-6} \mathrm{~m}$. The initial particles of the second phase are distributed randomly in a liquid phase. There are 16875 particles and their initial radius, $r_{0}$, is randomly set at $2.0 d x$ to $3.0 d x$. The initial composition of liquid, $c_{L}^{0}$, is provided through the dimensionless local supersaturation $u_{0}\left[\equiv\left(c_{L}^{0}-c_{L}^{e}\right) /\left(c_{L}^{e}-c_{S}^{e}\right)\right]$, and the composition of the second phase is determined through the local equilibrium condition. Two liquid compositions are considered: $u_{0}=-0.20$ and -0.30 . The temperature gradient, $G$, is applied to the system along the $x$-axis, and two temperature gradients are considered, $G=4.0 \times 10^{3}$ and $8.0 \times 10^{3} \mathrm{~K} / \mathrm{m}$, as well as a uniform temperature field $(G=0 \mathrm{~K} / \mathrm{m})$. For $G=0 \mathrm{~K} / \mathrm{m}$, a uniform temperature $T=850 \mathrm{~K}$ is applied to the system. For $G \neq 0 \mathrm{~K} / \mathrm{m}$, the temperature at the center of the system is fixed to $850 \mathrm{~K}$, where the temperature on the left side is set higher than that on the right side. For the sake of simplicity, the particle coalesces are not considered. Therefore, these values of temperature, composition, and temperature gradient are chosen such that the volume fraction of a particle does not exceed 0.4. ${ }^{28}$ The time step $d t$ is set to $1.5 \times 10^{-4} \mathrm{~s}$, and a time evolution process is investigated until $t_{\max }=750 \mathrm{~s}$. The interface width is set to $W=0.8 d x$. The zero-flux boundary condition (or Neumann boundary condition) is employed at the left and right edges of the system, while the periodic boundary condition is used at the upper and lower edges.

In addition to $2 \mathrm{D}$ simulations thus described, we carried out three-dimensional (3D) simulations for $G=0,4.0 \times 10^{3}$, and $8.0 \times 10^{3} \mathrm{~K} / \mathrm{m}$. We employed the computational domain of $6144 \times 192 \times 192 \mu \mathrm{m}^{3}$ with grid spacing $d x=d y=d z$ $=1.5 \times 10^{-6} \mathrm{~m}$. The temperature gradient is applied in the $x$-direction. The Neumann boundary is accordingly employed at the edges of $x=0$ and $6144 \mu \mathrm{m}$, while the periodic boundary condition is applied in the $y$-and $z$-directions. About 1000 particles are randomly distributed in the initial state. The values of $u_{0}$ and $k$ are set to -0.20 and 0.34 , respectively. The time step $d t$ is set to $1.1 \times 10^{-4} \mathrm{~s}$, and a time evolution process is investigated until $t_{\max }=536 \mathrm{~s}$. All other conditions are the same as those in the $2 \mathrm{D}$ simulations. All simulations in this study are accelerated by a graphics processing unit. 
A preliminary work is conducted to clarify the direction of solute diffusion between neighboring solid particles with and without temperature gradient (see the Appendix). The calculated results are consistent with those shown in Sec. III. They clearly show that the direction of solute diffusion in a non-uniform temperature field is quite different from that in a uniform temperature field.

\section{RESULTS AND DISCUSSION}

\section{A. Effects of temperature gradient on growth rate, migration velocity, and size distribution}

The initial particle distribution and time evolution process of microstructures calculated in uniform and non-uniform temperature fields $\left(G=0\right.$ and $\left.4.0 \times 10^{3} \mathrm{~K} / \mathrm{m}\right)$ for $u_{0}=-0.30$ and $k=0.34$ are presented in Figs. 1 and 2, respectively. In Fig. 1, all particles grow with time at the initial stage [see Fig. 1(b2)], but then only large particles continue growing and small particles start diminishing. This is typical Ostwald ripening behavior. When the temperature gradient is applied, the particle distribution becomes non-uniform along the $x$-axis [Figs. 2(a1)-2(a4)]. The particle density is low in the high temperature region (left side), while it is high in the low temperature region (right side). This non-uniformity becomes significant with time. Furthermore, each particle moves to the left side while exhibiting Ostwald ripening behavior [Figs. 2(b1)-2(b4)]. The migration behavior of particles toward the warmer region of the system is consistent with previous work by Snyder et al. ${ }^{19}$ The migration of a particle takes place by the migration of the solid-liquid interface. In the present system, this migration behavior can be attributed to the diffusion flux caused by the temperature dependence of solubility (see the Appendix). The solute diffusion induces the growth (dissolution) of particles on the left (right) side due to the differing solubility of solute at each temperature, which results in the movement of particles to the left side. Note that the circular shape of particles does not significantly deviate during their migration under the temperature gradient.

In Fig. 2, it can be seen that planar regions of liquid and solid phases are formed at high-temperature (left) and low-temperature (right) sides, respectively, even though the temperatures at both edges of the computational domain are in the range of two-phase equilibrium. This is due to the effect of the zero-flux boundary condition. Because of the zero-flux boundary condition, the solute atoms are accumulated (depleted) in the left (right) side, forming the liquid (solid) region. In the following analyses, therefore, only the two-phase region is considered while the effect of the zero-flux boundary condition is neglected; i.e., the planar regions of liquid and solid are removed. Specifically, the regions of 1572 and $2322 \mu \mathrm{m}$ are, respectively, eliminated from both left and right edges in the analyses for $G=4.0 \times 10^{3}$ and $8.0 \times 10^{3} \mathrm{~K} / \mathrm{m}$. This results in the same temperature range $(844-856 \mathrm{~K})$ of the analyzed system domains for $G=4.0 \times 10^{3}$ and $8.0 \times 10^{3} \mathrm{~K} / \mathrm{m}$.

In all cases, although not shown here, the number of particles monotonically decreases with time, while their average radius monotonically increases with time. The average area fraction of the second phase is almost independent of time after an initial transient period, and steady-state growth is achieved after $t=300 \mathrm{~s}\left(\equiv t_{c}\right)$ in all cases. The time dependence of the average radius of particles after $t=t_{c}$ is plotted in Fig. 3, where the horizontal axis is the time after $t=t_{c}$ and the vertical axis represents the cube of average particle radius,
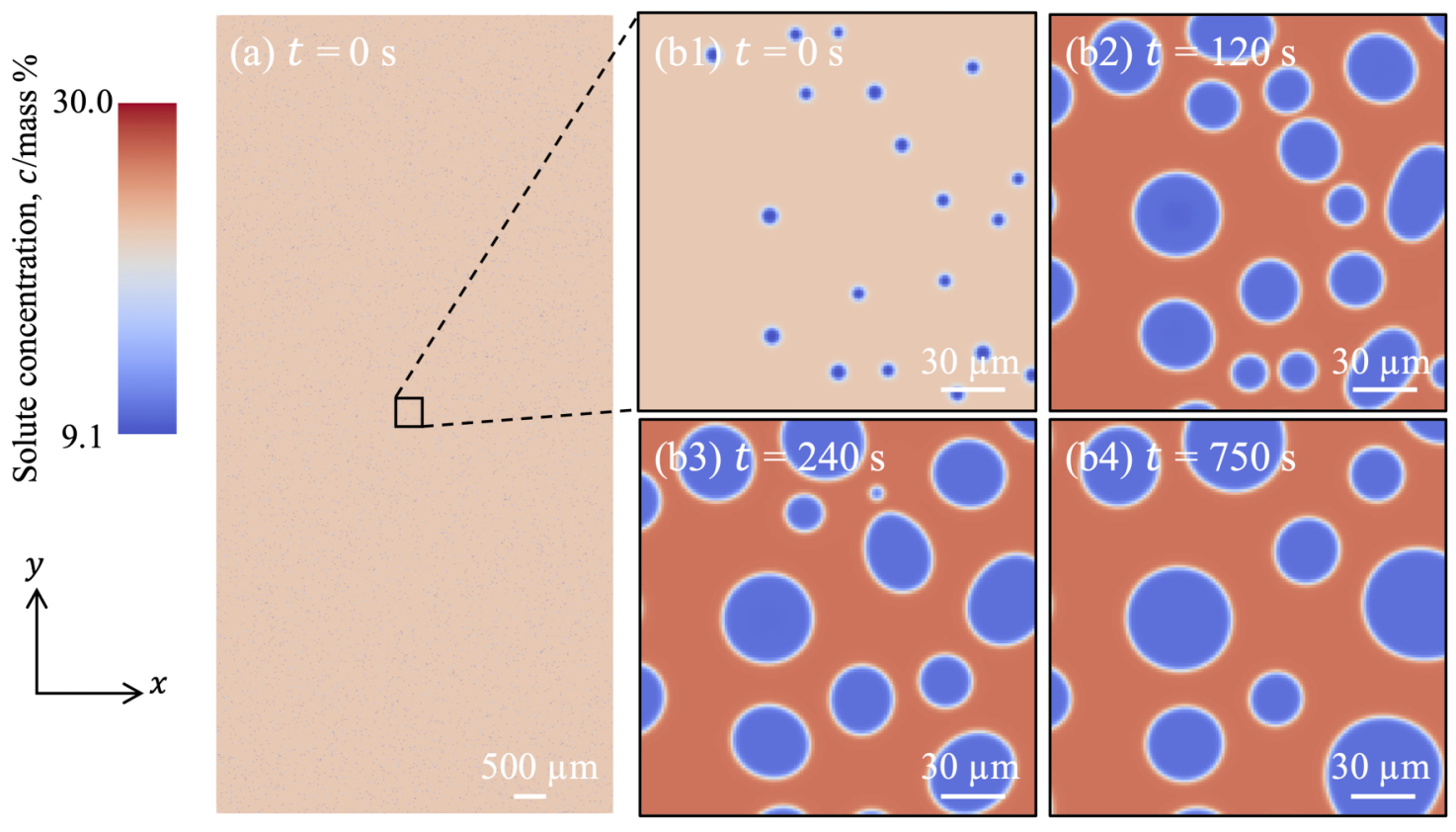

FIG. 1. Solute concentration map calculated for $u_{0}=-0.30, k=0.34$, and $G=0 \mathrm{~K} / \mathrm{m}$. (a) Initial microstructure, and (b1)-(b4) the microstructures at $t=0,120,240$, and $750 \mathrm{~s}$ in the small region indicated by a black square in (a). 

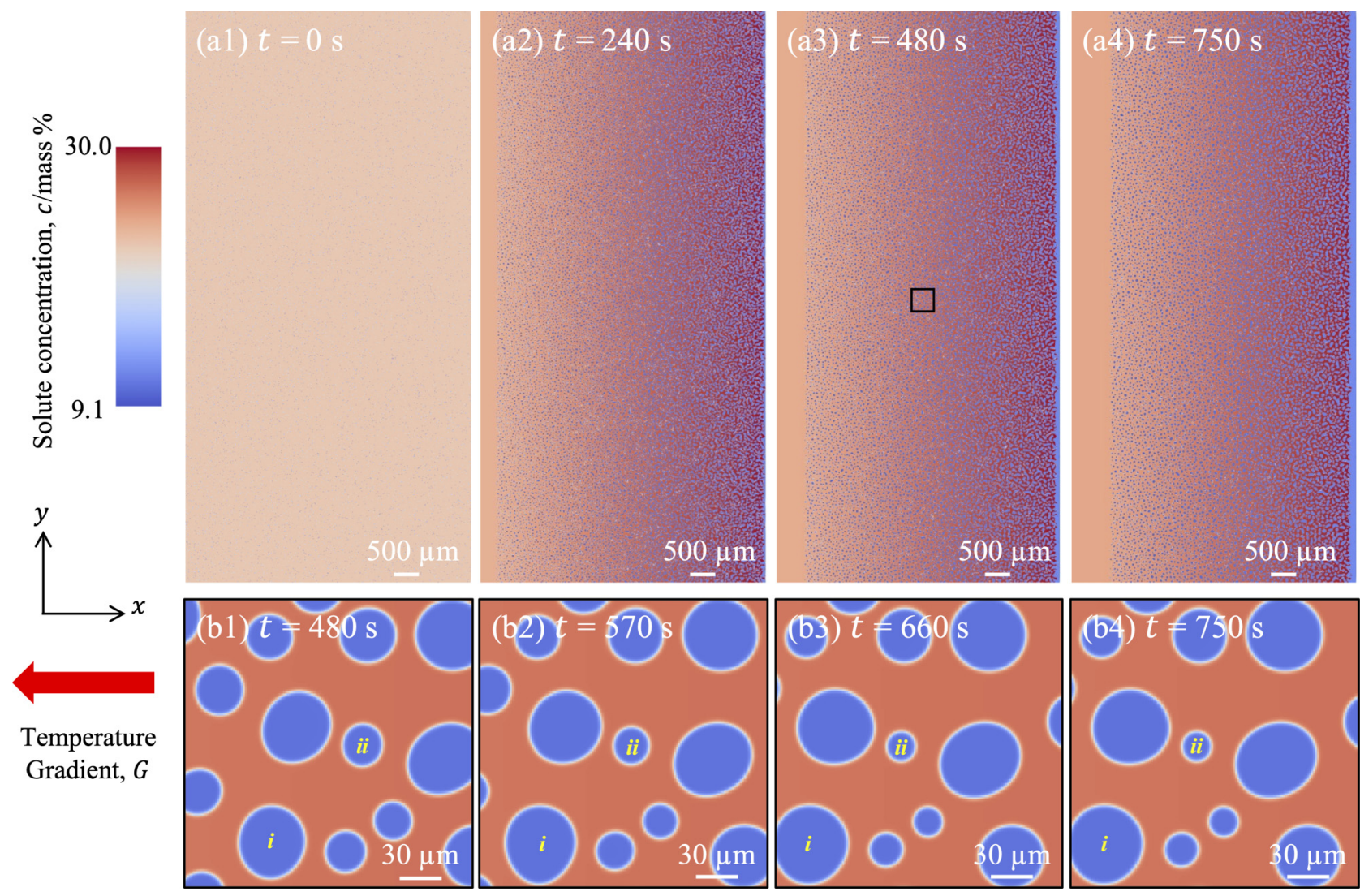

FIG. 2. Calculated time evolution process in the temperature gradient $\left(G=4.0 \times 10^{3} \mathrm{~K} / \mathrm{m}\right)$ for $u_{0}=-0.30$ and $k=0.34$. (a1)-(a4) are the solute concentration profiles at $t=0,240,480$, and $750 \mathrm{~s}$, respectively, in the whole system, and (b1)-(b4) are magnified profiles in the small region indicated by a black square in (a3). As seen in (b1)(b4), all particles move to the higher temperature side (or the left side) with time, and large particles (see particle i) grow at the expense of small particles (see particle ii).

$r^{3}-r_{c}^{3}$. One can see that the cube of the average particle radius is proportional to the time for both uniform and non-uniform temperature fields, and the growth rate of particles significantly increases with the magnitude of the temperature gradient.

The effects of $u_{0}$ and $k$ on the coarsening behaviors are investigated. Figure 4 shows the temporal changes of average particle radius calculated for $u_{0}=-0.20$ and -0.30 and $k=0.14$ and 0.34 in uniform and non-uniform temperature fields, $G=0$ and $4.0 \times 10^{3} \mathrm{~K} / \mathrm{m}$. The growth rate is faster for $u_{0}=-0.30$ than for $u_{0}=-0.20$. This faster coarsening behavior for $u_{0}=-0.30$ is due to the fact that the area fraction of the particles is increased as $u_{0}$ decreases in the alloy system under consideration. The increment of area fraction of particles causes a small diffusion distance between neighboring particles. As for the effect of $k$, the growth rates for $k=0.34$ are higher than those for $k=0.14$. This can be explained from the following balance equation at the solid-liquid interface:

$$
\left(c_{L}^{*}-c_{S}^{*}\right) v=-\left.D_{L} \frac{d c}{d x}\right|_{i}
$$

where $v$ is the velocity of the interface, $c_{L}^{*}\left(c_{S}^{*}\right)$ is the liquid (solid) concentration at the interface, and $d c /\left.d x\right|_{i}$ is the concentration gradient at the interface in the direction normal to the interface. Because the difference in concentrations between solid and liquid phases at the interface is small for a large $k\left(\equiv c_{S}^{e} / c_{L}^{e}\right)$, the interface velocity increases when the concentration gradient is not significantly altered by a different value of $k$.

A ratio of the growth rate constants of uniform and nonuniform temperature fields, $K_{\text {het }} / K_{\text {hom }}$, is calculated for $u_{0}=-0.20$ and -0.30 with $k=0.34$. It is found that $K_{\text {het }} / K_{\text {hom }} \approx 1.22$ for both alloy compositions. Also, in the case of $k=0.14$, it is found that $K_{\text {het }} / K_{\text {hom }} \approx 1.13$ for different values of $u_{0}$. This suggests the possibility that the difference in growth rates between uniform and nonuniform temperature fields is not sensitive to alloy composition.

The temporal changes of particle size distribution after $t=t_{c}$ are examined, and the calculated results for different temperature gradients are shown in Figs. 5(a)-5(c), where the horizontal axes represent the particle radius $r$ normalized by the average value $\langle r\rangle$. In order to increase the sample data, the same simulations with different initial particle distributions were conducted seven times and the averaged data are plotted. One can see that for all temperature 


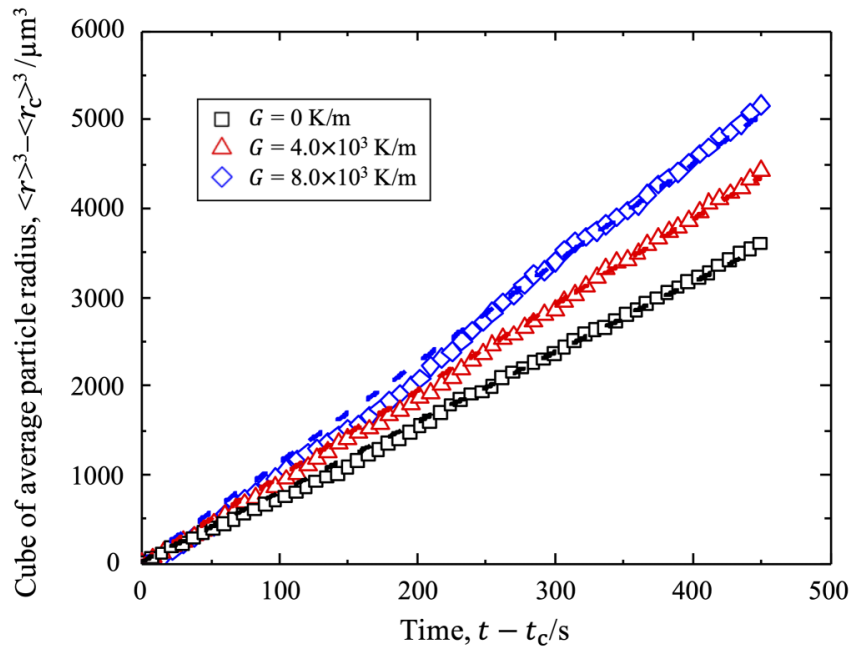

FIG. 3. Temporal change of average radius of particles calculated for $u_{0}=-0.30$ and $k=0.34$ in various temperature gradients: $G=0$ (black), $4.0 \times 10^{3}$ (red), and $8.0 \times 10^{3}$ (blue) $\mathrm{K} / \mathrm{m}$. The dashed lines represent the approximated linear functions with growth-rate constants $K=7.9,8.6$, and $11.2 \mu \mathrm{m}^{3} / \mathrm{s}$ for $\mathrm{G}=0,4.0 \times 10^{3}$, and $8.0 \times 10^{3} \mathrm{~K} / \mathrm{m}$, respectively.

gradients, the peak positions decrease and shift to the larger particle radius region with time. Importantly, the size distributions exhibit a steady-state distribution; i.e., self-similarity. Although not discussed here, these steady-state size distributions cannot be explained by the theoretical models proposed for a uniform temperature field. ${ }^{4,28,29}$ In Fig. 5(d), the calculated size distributions for different temperature gradients are compared at $t-t_{c}=450 \mathrm{~s}$. The size distribution becomes broader as the temperature gradient increases. The reason for this broad size distribution for large temperature gradients is discussed in Sec. III B, as is the reason for the high growth rate of particles for large temperature gradients.

\section{B. Analysis of local coarsening behavior and migration process}

The coarsening behavior and migration process of particles are given a closer look by dividing the simulation system in the non-uniform temperature field $\left(G=4.0 \times 10^{3} \mathrm{~K} / \mathrm{m}\right)$ into four temperature regions: (I) $853-856$, (II) $850-853$, (III) $847-850$, and (IV) $844-847 \mathrm{~K}$.

The calculated temporal changes of average radius in four temperature regions are shown in Fig. 6(a). The time evolution process of the entire system corresponds to that shown by triangle plots in Fig. 3. The cube of the average radius of particles is proportional to the time in each temperature region. The coarsening occurs rapidly in the low temperature regions. The growth rate largely depends on local temperature and, thereby, particles of largely different sizes exist in a non-uniform temperature field. This explains the broad particle size distribution for large temperature gradients in Fig. 5(d).
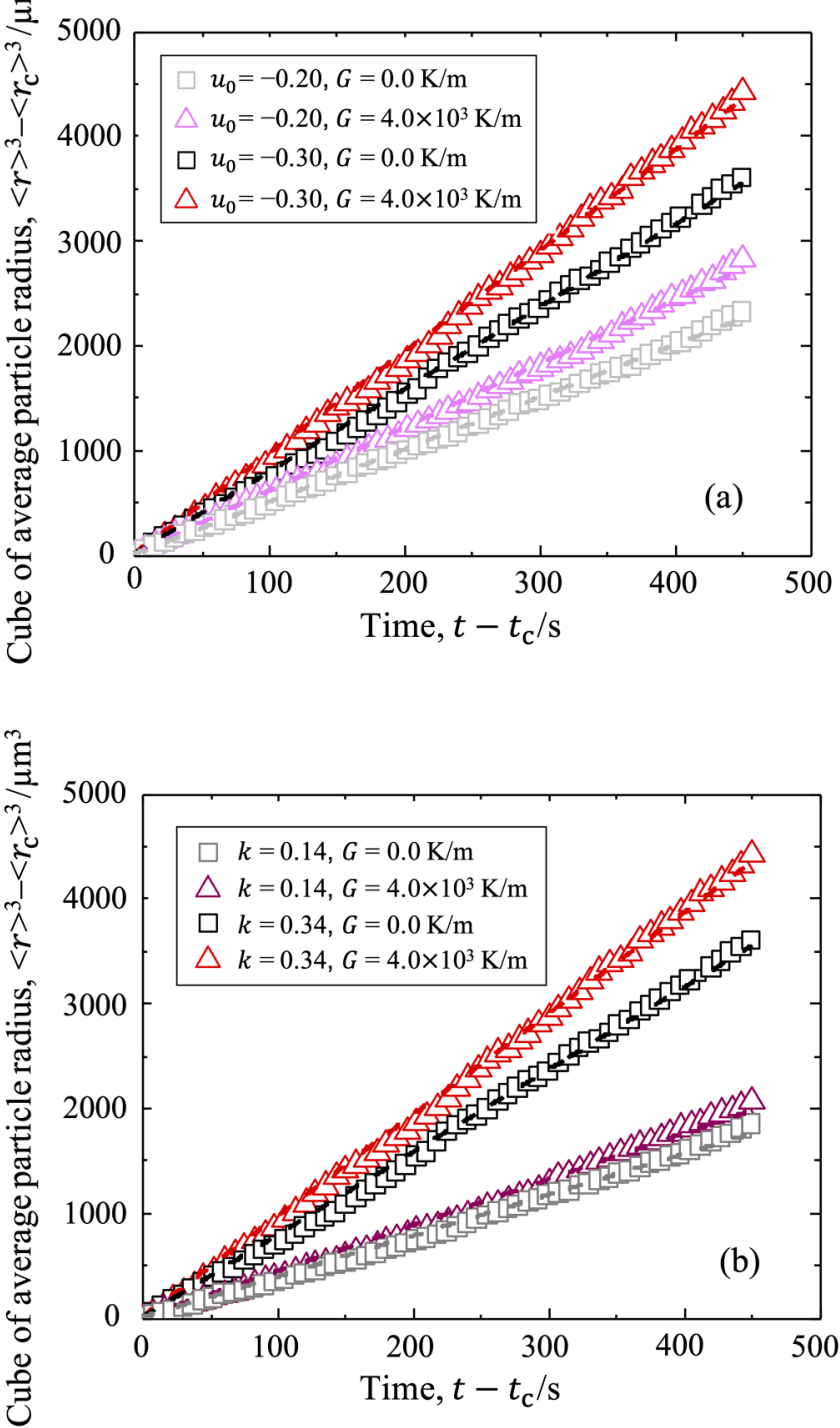

FIG. 4. Temporal change of average particle radius for $G=0$ and $4.0 \times 10^{3} \mathrm{~K} / \mathrm{m}$ after $t=t_{c}$, using (a) $u_{0}=-0.20$ and -0.30 with $k=0.34$ and (b) $k=0.14$ and 0.34 with $u_{0}=-0.30$

Our simulations showed that the concentration, averaged over the local region, is constant in time and space during coarsening in the temperature gradient. Therefore, the difference in local growth rate may be attributed to the different area fraction. The temporal changes of area fraction in the four regions are shown in Fig. 6(b). The area fraction of particles in the low temperature region is larger than that in the high temperature region. This is readily understood from the lever rule in the phase equilibria of our focus. As the area fraction increases, the inter-particle distance (i.e., the diffusion distance between particles) decreases. Therefore, the large 

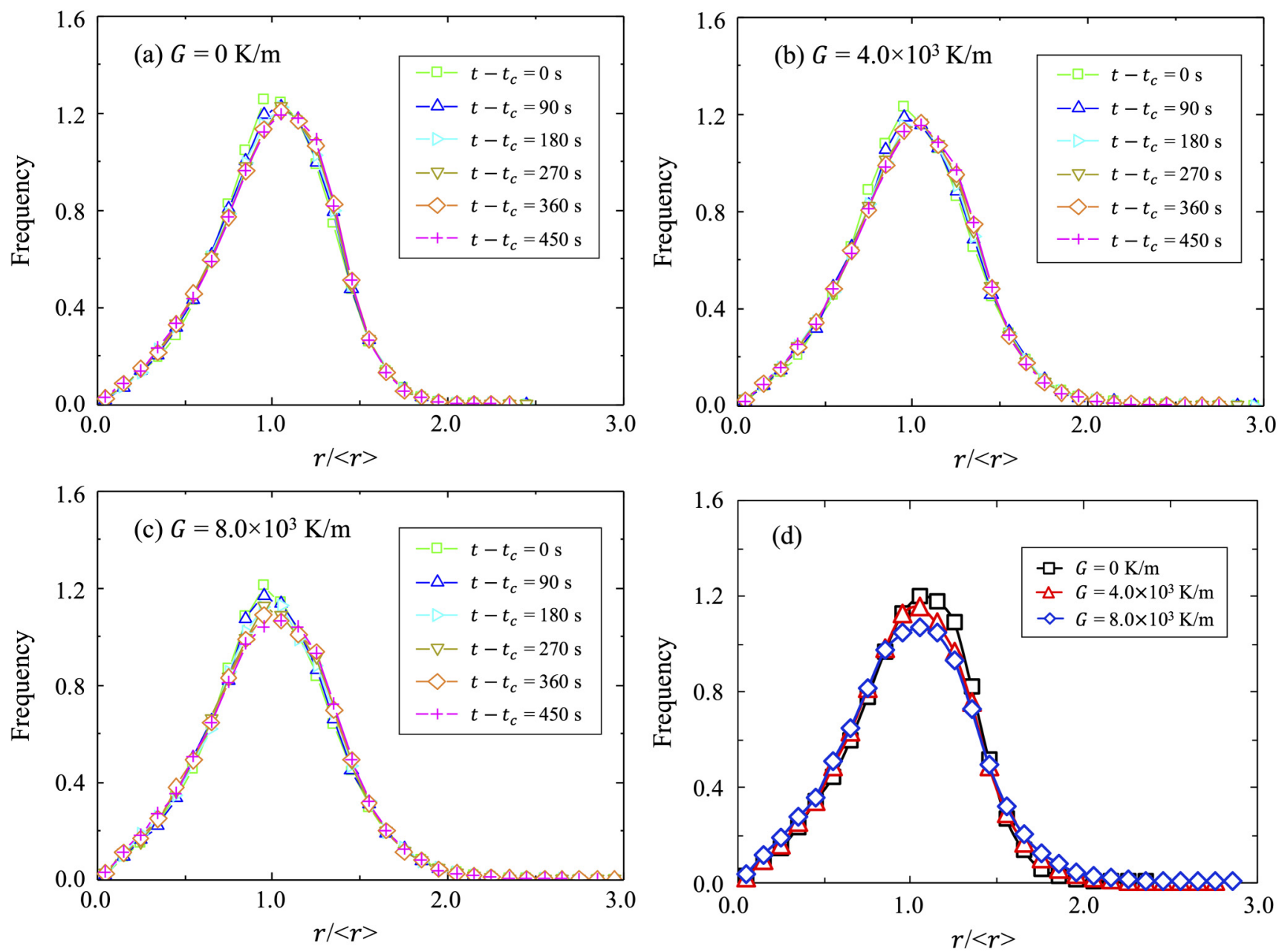

FIG. 5. Temporal change of particle size distribution after $t=t_{c}$ calculated for $u_{0}=-0.30$ and $k=0.34$ in various temperature gradients: $G=$ (a) 0, (b) $4.0 \times 10^{3}$, and (c) $8.0 \times 10^{3} \mathrm{~K} / \mathrm{m}$. (d) Comparison of the calculated particle size distributions at $t-t_{c}=450 \mathrm{~s}$ using the three different temperature gradients.

area fraction of particles is one of the reasons for the high growth rate at low temperatures in Fig. 6(a). However, it is not the only reason. If the area fraction were the only reason for the high growth rate, the growth rate averaged over the whole system should be the same, regardless of the value of the temperature gradient when the maximum and minimum temperatures are the same in the system under analysis. In our analysis, the maximum (left) and minimum (right) temperatures are set to the same values in the cases of $G=4.0 \times 10^{3}$ and $8.0 \times 10^{3} \mathrm{~K} / \mathrm{m}$. However, the growth rate averaged over the whole system increases with the temperature gradient, as shown in Fig. 3. Hence, there must be another important factor that accelerates the coarsening process in the temperature gradient. It is particle migration, as detailed below.

The dependence of individual particle velocity on its size is investigated and is shown in Fig. 7(a). These are the velocities of each particle averaged over $t-t_{c}=442.5-450 \mathrm{~s}$. No correlations between particle size and velocity can be found. This indicates that the migration velocity is predominantly determined by the diffusion flux in the matrix driven by the temperature dependence of solubility. The time dependence of migration velocity averaged in each temperature region, $\bar{v}$, is shown in Fig. 7(b). The particles continue to move from low to high temperature regions over the entire time period. In addition, it can be seen that the average velocities of particles are higher at high temperatures than at low temperatures. These high velocities of particles at high temperatures stem from a small difference in the solute concentrations of the solid and liquid phases. When the right-hand side of Eq. (7) is nearly constant, the velocity of the solid-liquid interface increases as the difference between solute concentrations of solid and liquid decreases. Therefore, the migration velocity of particles is faster at 

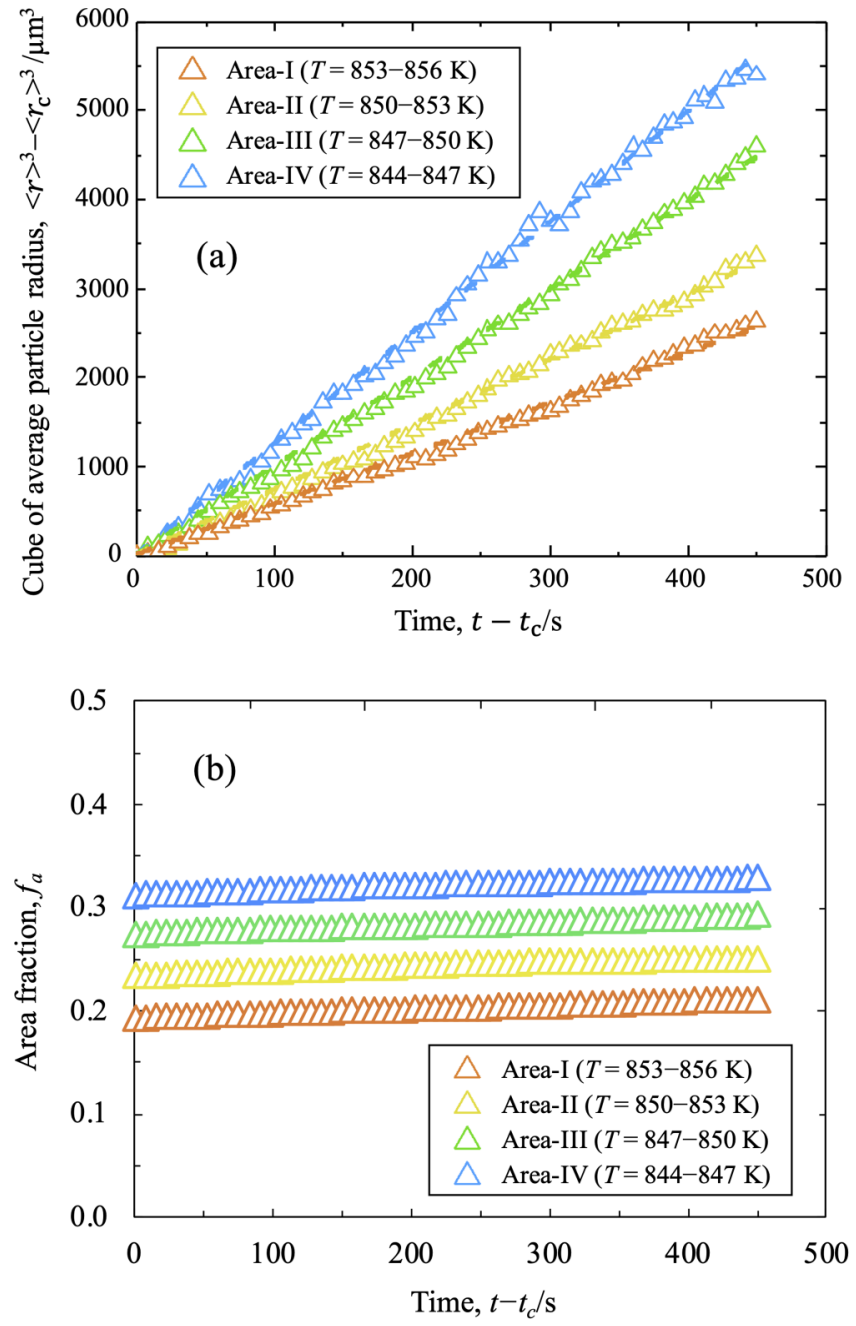

FIG. 6. Temporal change of (a) average radius of particles and (b) area fraction in each local area after $t=t_{c}$ calculated for $u_{0}=-0.30, k=0.34$, and $G=4.0 \times 10^{3} \mathrm{~K} / \mathrm{m}$. The dashed lines in (a) represent approximated linear functions with the growth rate constants $K=5.7,7.3,9.9$, and $12.4 \mu \mathrm{m}^{3} / \mathrm{s}$ for area-I, -II, -III, and -IV, respectively.

high temperatures than at low temperatures. Note that $D_{L}$ is temperature-dependent, as seen in Table I, and may affect the interface velocity $v$. However, we checked its influence on velocity by fixing $D_{L}$ to a constant value and found that its effect is negligible.

As described above, the high area fraction of the particles yields the high growth rate at low temperature. The relatively large particles grown at low temperature then moves toward the high temperature region. Such migration of large particles increases the average radius in the high temperature region. When the temperature gradient is high, the migration velocity increases in the entire system. Many large particles grown in the
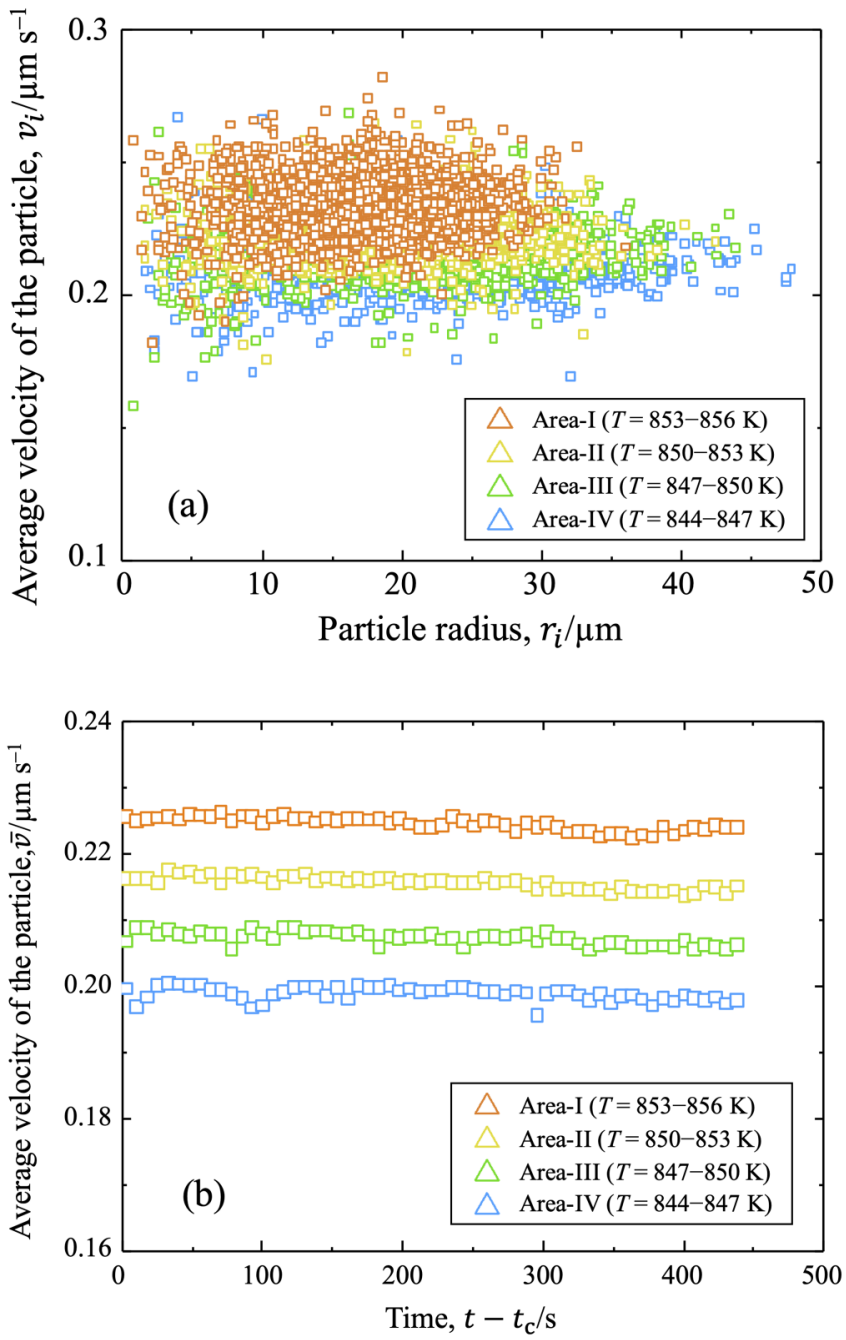

FIG. 7. (a) Velocity of each particle, $v_{i}$, averaged over $t-t_{c}=442.5-450 \mathrm{~s}$ in each local region calculated for $u_{0}=-0.30, k=0.34$, and $G=4.0 \times 10^{3} \mathrm{~K} / \mathrm{m}$. The horizontal axis represents the particle radius, $r_{i}$. (b) Temporal change of migration velocity averaged in each local region after $t=t_{c}$ calculated for $u_{0}$ $=-0.30, k=0.34$, and $\mathrm{G}=4.0 \times 10^{3} \mathrm{~K} / \mathrm{m}$.

low temperature region move to the high temperature region, causing an apparent acceleration of the growth rate of particles in the entire system.

\section{3D analysis}

The coarsening processes in the $2 \mathrm{D}$ system are investigated in Secs. III A and III B. Here, 3D simulations are conducted to confirm whether or not the same behavior as with the $2 \mathrm{D}$ simulations can be obtained, e.g., an acceleration of growth rate with temperature gradient and a migration of second-phase particles to the warmer temperature side. 

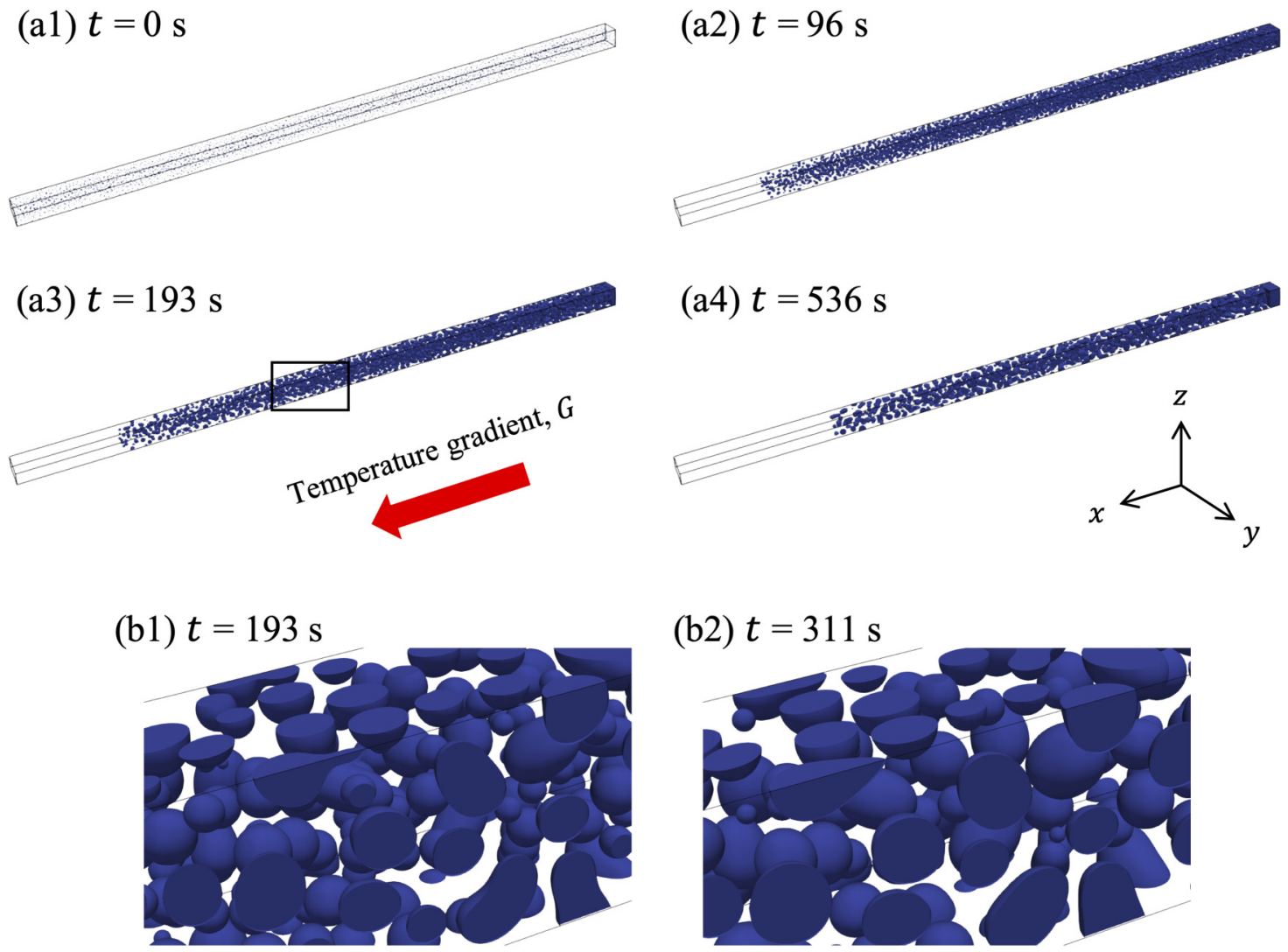

(b2) $t=311 \mathrm{~s}$

(b3) $t=429 \mathrm{~s}$
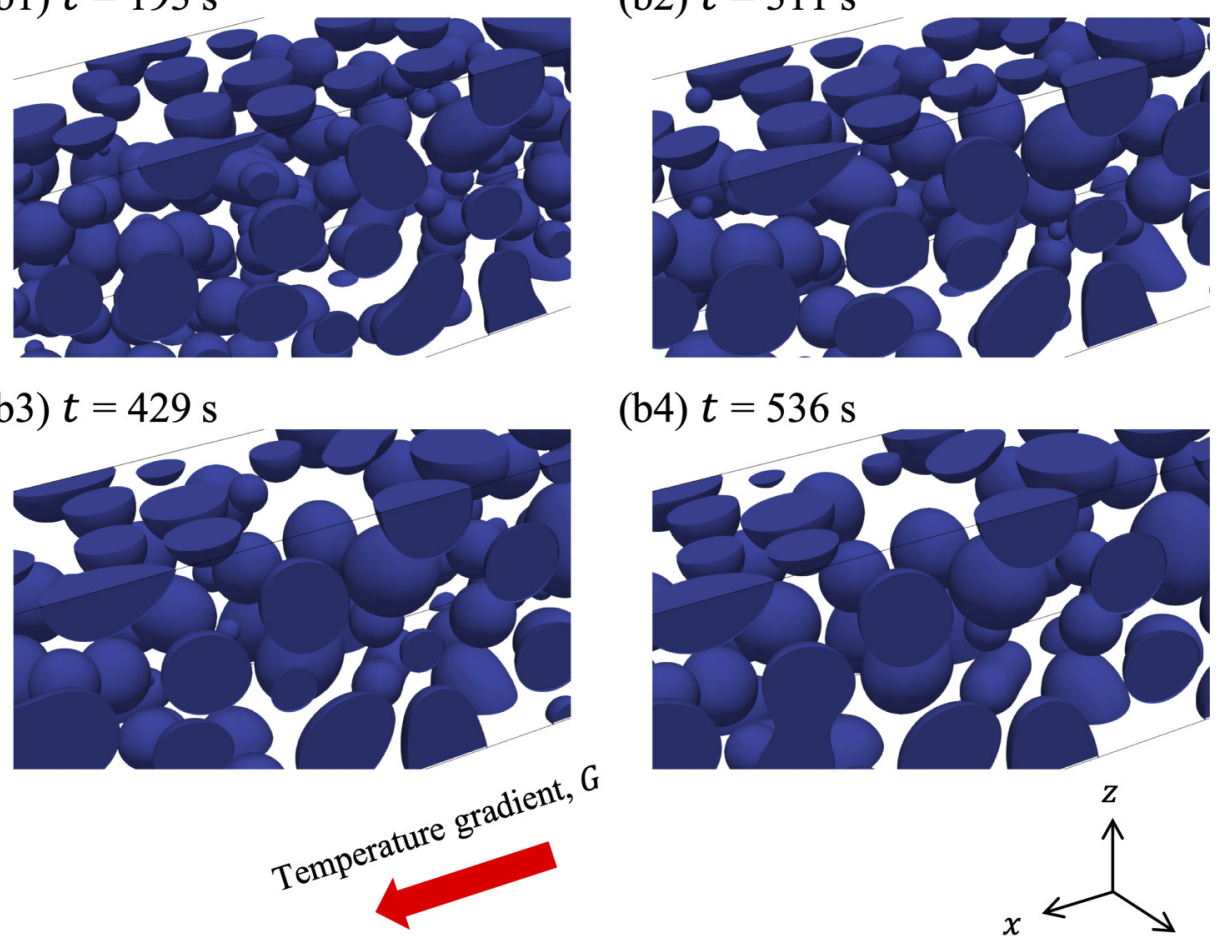

(b4) $t=536 \mathrm{~s}$
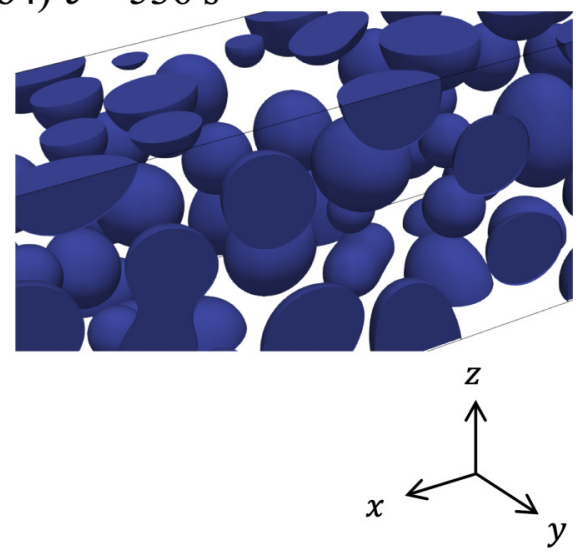

FIG. 8. Time evolution process of 3D microstructure under the temperature gradient $\left(G=4.0 \times 10^{3} \mathrm{~K} / \mathrm{m}\right)$ in (a) the whole system and (b) a part of the system. The magnified part (b) is of the region indicated by a black square in (a3). The second-phase particles are colored in blue and the liquid phase is made transparent.

Figure 8 shows the time evolution process of the $3 \mathrm{D}$ microstructure under a temperature gradient $\left(G=4.0 \times 10^{3} \mathrm{~K} / \mathrm{m}\right)$. In Figs. 8(b1)-8(b4), one finds that the second-phase particles migrate from low (right) to high (left) temperature regions while exhibiting Ostwald ripening, as seen in the $2 \mathrm{D}$ simulations. This is ascribed to the diffusion flux due to the temperature dependence of solute solubility, as mentioned in Sec. III A. The formation of liquid and solid regions can be seen at the left and right edges of the system, respectively [Figs. 8(a1)-8(a4)]. This is due to the zeroflux boundary condition. In order to eliminate the effect on the 

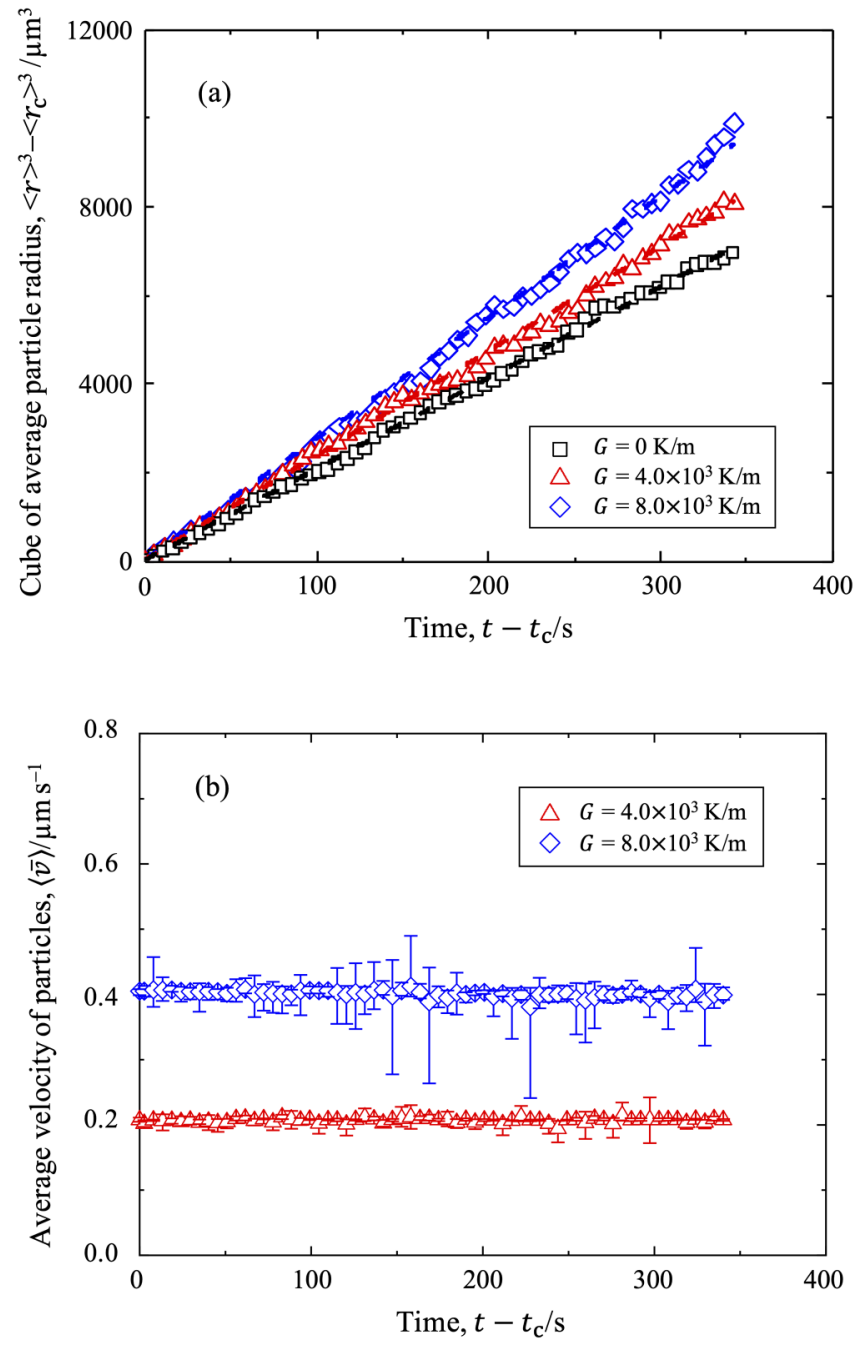

FIG. 9. Temporal change of (a) average radius and (b) average velocity of particles after $t=t_{c}$ in 3D simulations. The dashed lines in (a) represent approximated linear functions with the growth rate constants $K=20.5,23.5$, and $27.3 \mu \mathrm{m}^{3} / \mathrm{s}$ for $G=0,4.0 \times 10^{3}$, and $8.0 \times 10^{3} \mathrm{~K} / \mathrm{m}$, respectively.

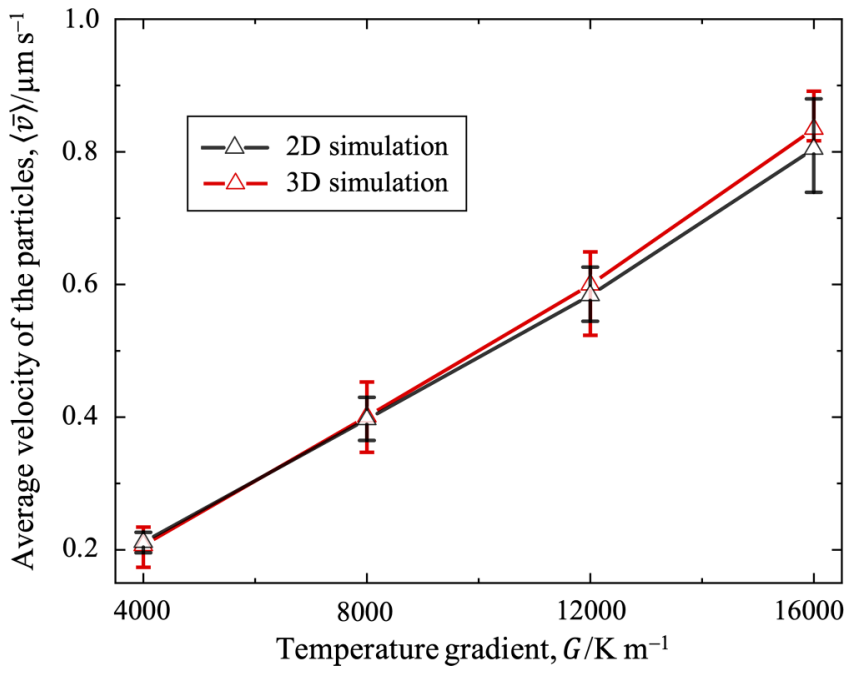

FIG. 10. The dependence of the average migration velocity of a particle on the temperature gradient calculated for $u_{0}=-0.20$ and $k=0.34$ in the $2 \mathrm{D}$ and $3 \mathrm{D}$ simulations. The plots represent averaged values over the results of several simulations having different initial conditions.

following analysis, the regions of $2322 \mu \mathrm{m}(2697 \mu \mathrm{m})$ from the left and right edges are removed for $G=4.0 \times 10^{3} \mathrm{~K} / \mathrm{m}\left(8.0 \times 10^{3} \mathrm{~K} / \mathrm{m}\right)$. Note that the analyzed system domains for $G=4.0 \times 10^{3}$ and $8.0 \times 10^{3} \mathrm{~K} / \mathrm{m}$ are in the same temperature range $(T=847-853 \mathrm{~K})$.

To investigate the effects of the magnitude of temperature gradient on the coarsening process, the same simulation with different initial particle distributions was conducted four times for $G=0$ or $4.0 \times 10^{3} \mathrm{~K} / \mathrm{m}$ and eight times for $G=8.0 \times 10^{3} \mathrm{~K} / \mathrm{m}$, in order to increase the sample data. The calculated temporal changes of average radius and velocity of particles are shown in Figs. 9(a) and 9(b), respectively. In these figures, the horizontal axes indicate the time after $t=193 \mathrm{~s}\left(\equiv t_{c}\right)$ because the steady-state growth was confirmed from this time period. In Fig. 9(a), the cube of average particle radius is proportional to time in all cases, and the growth rate of particles increases with the temperature gradient. Moreover, as can be seen in Fig. 9(b), the average velocity of particles is constant during the coarsening process under the temperature gradient. These results are consistent with those in the $2 \mathrm{D}$ simulations. The particle size

TABLE II. Calculation conditions of additional simulations for 2D and 3D systems.

\begin{tabular}{lcccc}
\hline \hline & $2 \mathrm{D}$ & & & \\
\hline Temperature gradient, $G(\mathrm{~K} / \mathrm{m})$ & $12.0 \times 10^{3}$ & $16.0 \times 10^{3}$ & $12.0 \times 10^{3}$ & $16.0 \times 10^{3}$ \\
System size $\left(\mu \mathrm{m}^{2}\right.$ or $\left.\mu \mathrm{m}^{3}\right)$ & $4096 \times 8192$ & $2048 \times 4096$ & $4096 \times 128 \times 128$ & $2048 \times 64 \times 64$ \\
Grid size, $d x(\mathrm{~m})$ & $1.0 \times 10^{-6}$ & $0.5 \times 10^{-6}$ & $1.0 \times 10^{-6}$ & $0.5 \times 10^{-6}$ \\
Time step, $d t(\mathrm{~s})$ & $6.7 \times 10^{-5}$ & $1.7 \times 10^{-5}$ & $4.8 \times 10^{-5}$ & $1.2 \times 10^{-5}$ \\
Retention time, $t_{\max }(\mathrm{s})$ & 510 & 150 & 238 & 42 \\
\hline
\end{tabular}


distribution cannot accurately be obtained in this 3D analysis because of an insufficient number of particles in the computational domain. It remains to be investigated in a future work.

The average migration velocities of particles obtained from the $2 \mathrm{D}$ and $3 \mathrm{D}$ simulations are plotted with respect to the temperature gradient in Fig. 10, where additional simulations are performed at very high temperature gradients, $G=12.0 \times 10^{3}$ and $16.0 \times 10^{3} \mathrm{~K} / \mathrm{m}$, with $u_{0}=-0.20$ and $k=0.34$. The calculation conditions of the additional simulations are summarized in Table II. From Fig. 10, it can be seen that the velocity increases almost linearly with the temperature gradient in both $2 \mathrm{D}$ and $3 \mathrm{D}$ simulations. This is consistent with the discussion in the early works. ${ }^{15}$ The average migration velocities calculated from the $2 \mathrm{D}$ and $3 \mathrm{D}$ simulations show a quantitative agreement. This suggests that the migration behavior of particles is entirely attributable to the diffusion flux driven by the concentration gradient originating from the temperature dependence of solute solubility. It is noteworthy that the velocity becomes as high as $0.8 \mu \mathrm{m} / \mathrm{s}$ when $G$ is $16.0 \times 10^{3} \mathrm{~K} / \mathrm{m}$. Therefore, the migration behavior of particles cannot be negligible when the material is subjected to an extended period of annealing under a high-temperature gradient.

\section{CONCLUSION}

The effects of a temperature gradient on the coarsening behaviors of a second phase distributed in a matrix were investigated using a quantitative phase-field model. It was confirmed that large particles grow with time at the expense of small particles in both uniform and non-uniform temperature fields. Regardless of the value of the temperature gradient, the cube of the particle radius is proportional to the time, and the particle size distribution shows a self-similarity when a system reaches a steady state. Importantly, the growth rate of particles increases with the temperature gradient and the steady-state size distribution depends on the temperature gradient. Moreover, a migration of particles from low to high temperature regions was observed during the coarsening process when a non-uniform temperature field is applied. While the high area fraction of the particles yields the high growth rate at low temperature, the relatively large particles grown at low temperature move toward the high temperature region. Hence, the migration of large particles results in an increase of average radius in the high temperature region and thereby in the whole system.

For the sake of simplicity, we focused on the process with a low volume fraction of particles to avoid particle coalesces. For a system with a larger volume fraction, the use of a multi-phase-field model would be required, as in Refs. 10 and 11. This is beyond the scope of the present research and remains as a future work. It is noteworthy that Ostwald ripening has been investigated using the phase-field crystal (PFC) method as well. ${ }^{30,31}$ The PFC method enables one to simulate the evolution of the microstructure at diffusive time scales and incorporate elasticity, multiple crystal orientations, grain boundaries, and dislocations, which are not considered in the present work. Although a system size used in the PFC method is still limited, it has a potential to elucidate the mechanism of Ostwald ripening from the atomistic point of view.

\section{ACKNOWLEDGMENTS}

This work was partially supported by KAKENHI, Grant-in-Aid for Research Activity Start-up, No. 20K22456, and Grant-in-Aid for Scientific Research (B), No. 19H02486, from the Japan Society for the Promotion of Science (JSPS).

\section{APPENDIX: THE EFFECT OF TEMPERATURE GRADIENT ON SOLUTE DIFFUSION DIRECTION}

Before elucidating the effect of temperature gradients on Ostwald ripening behavior, a preliminary work is conducted in this appendix, where the direction of solute diffusion between solid particles with and without a temperature gradient is investigated. A two-dimensional simulation size of $336 \times 96 \mu \mathrm{m}^{2}$ is considered here with $G=0$ and $4.0 \times 10^{3} \mathrm{~K} / \mathrm{m}$. Four solid particles of initial radius $r_{0}=24.0 d x(\mathrm{P} 1, \mathrm{P} 2, \mathrm{P} 4$, and $\mathrm{P} 5)$ and one with $r_{0}=2.0 d x$ (P3) are placed in a liquid phase as shown in Fig. 11. The temporal changes of the solute concentration profiles calculated for $u_{0}=-0.10$ and $k=0.34$ with and without a temperature gradient are shown in Fig. 12. It is found that particles migrate from the low temperature region to the high temperature region while exhibiting Ostwald ripening behavior. (The mechanism of this migration behavior is discussed in Secs. III A and III B.) It is noteworthy that the time at which the small solid particle (P3) disappears is the same in both calculations with and without a temperature gradient $(t=54.0 \mathrm{~s})$.

To clarify the effect of temperature gradient on solute diffusion, the temporal change of solute concentration profiles at $y=48 \mu \mathrm{m}$ (represented as red broken lines in Fig. 12) is shown in Fig. 13, where a low (high) concentration region corresponds to a solid (liquid) phase. In both simulations with and without a temperature gradient, the region of the solid particle P3 narrows with time and eventually becomes a liquid phase. However, the mechanisms of the transition of $\mathrm{P} 3$ to a liquid phase are quite different in the two cases. As can be seen in Figs. 13(a2) and 13(b2), there are concentration gradients from P2 and P4 to P3 for the uniform temperature field, while there is a concentration gradient only from $\mathrm{P} 4$ to P3 for the non-uniform temperature field. These results indicate that the solute diffuses from both $\mathrm{P} 2$ and $\mathrm{P} 4$ regions into the P3 region in a uniform temperature field, while solute diffusion occurs only from P4 into P3 in the non-uniform temperature field. This suggests that the direction of a solute flux is significantly influenced by the temperature gradient.

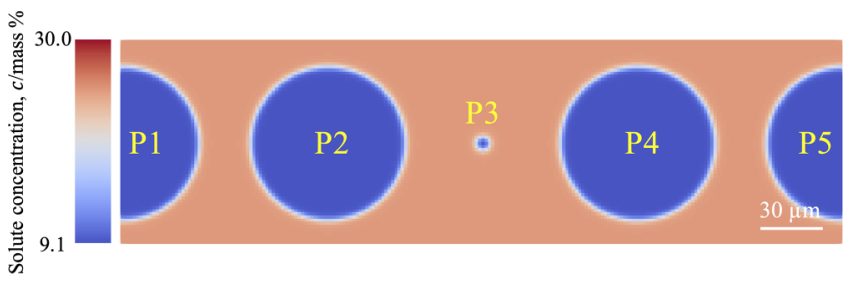

FIG. 11. Initial solute concentration profile. The large particles are denoted as $\mathrm{P} 1, \mathrm{P} 2$, P4, and P5. The small particle is denoted as P3. 

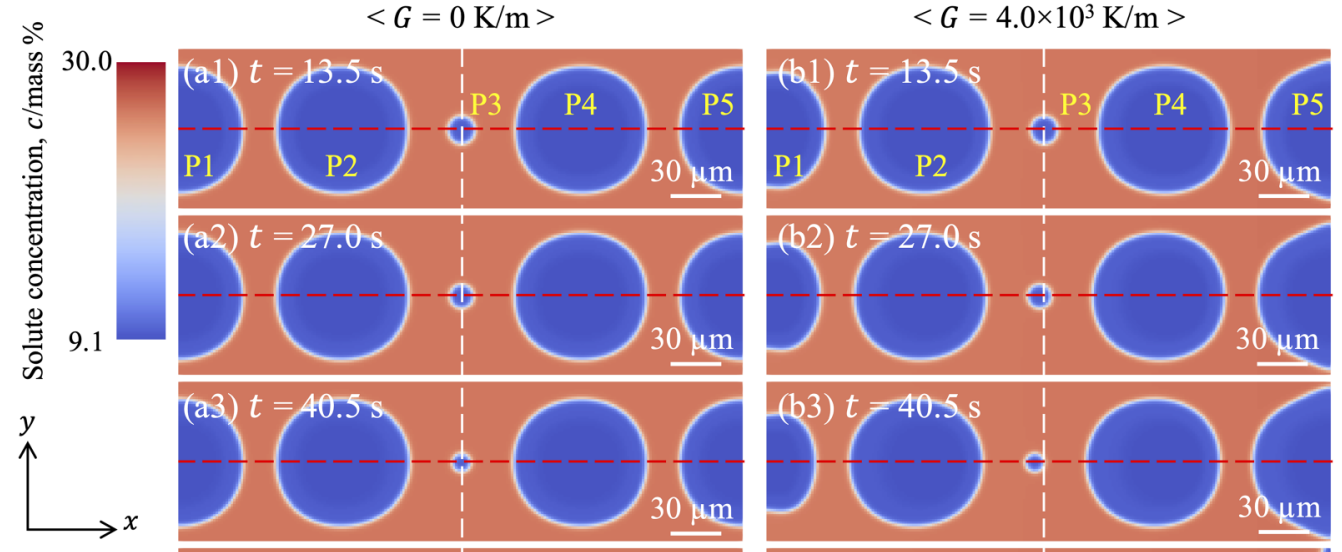

Temperature Gradient, $G$
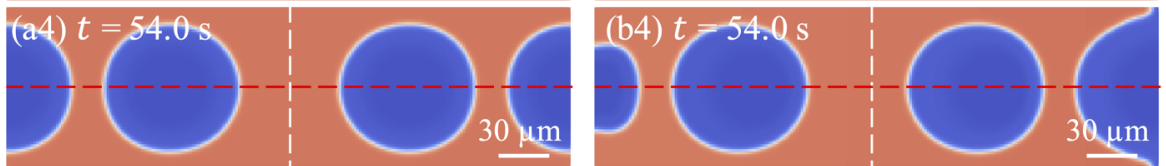

FIG. 12. Temporal changes of solute concentration map calculated for $u_{0}=-0.10$ and $k=0.34$ at $t=13.5,27.0,40.5$, and $54.0 \mathrm{~s}$ with (a) $\mathrm{G}=0$ and (b) $\mathrm{G}=4.0 \times 10^{3} \mathrm{~K} / \mathrm{m}$. For the uniform temperature field, the temperature is set to $850 \mathrm{~K}$. For the non-uniform temperature field, the temperature at the center of the system is set to $850 \mathrm{~K}$.
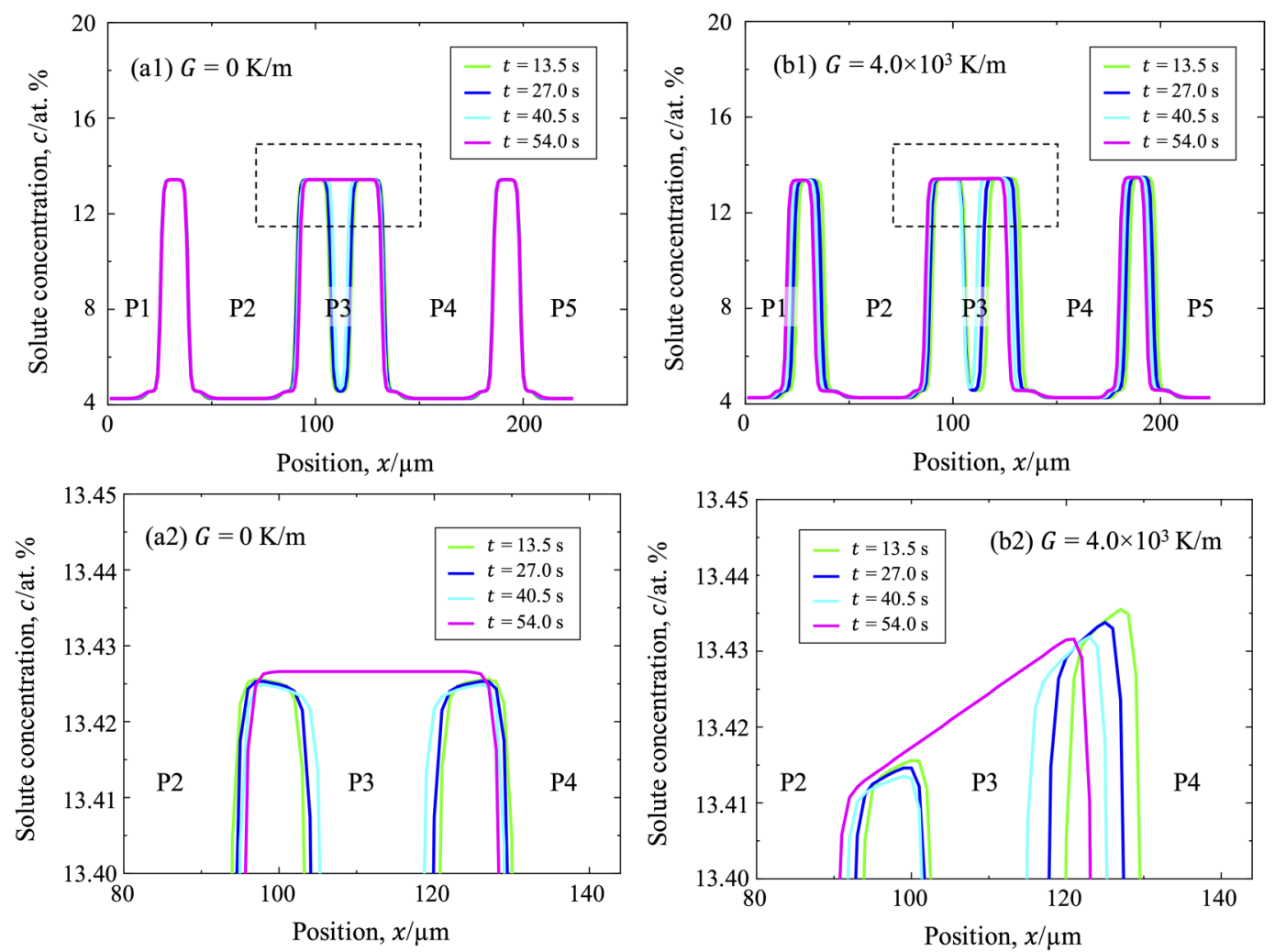

FIG. 13. Temporal changes of solute concentration profiles at $y=48 \mu \mathrm{m}$ (represented as red broken lines in Fig. 12). (a2) and (b2) are the magnified parts of (a1) and (b1), respectively. 


\section{DATA AVAILABILITY}

The data that support the findings of this study are available from the corresponding author upon reasonable request.

\section{REFERENCES}

${ }^{1}$ H. I. Aaronson, M. Enomoto, and J. K. Lee, Mechanisms of Diffusional Phase Transformations in Metals and Alloys (CRC Press, 2010).

${ }^{2}$ M. Hitchcock, Y. Wang, and Z. Fan, Acta Mater. 55, 1589 (2007).

${ }^{3}$ R. E. Smallman and A. H. W. Ngan, Modern Physical Metallurgy, 8th ed. (Butterworth-Heinemann, 2014), p. 499.

${ }^{4}$ L. M. Lifshitz and V. V. Slyozov, J. Phys. Chem. Solids 19, 35 (1961).

${ }^{5}$ C. Wagner, Z. Electrochem. 65, 581-591 (1961).

${ }^{6}$ T. M. Rogers and R. C. Desai, Phys. Rev. B 39, 11956 (1989).

${ }^{7}$ N. Akaiwa and P. W. Voorhees, Phys. Rev. E 49, 3860 (1994).

${ }^{8}$ V. A. Snyder, J. Alkemper, and P. W. Voorhees, Acta Mater. 49, 699-709 (2001).

${ }^{9}$ D. Fan, S. P. Chen, L.-Q. Chen, and P. W. Voorhees, Acta Mater. 50, 1895-1907 (2002).

${ }^{10}$ S. G. Kim, Acta Mater. 55, 6513-6525 (2007).

${ }^{11} \mathrm{~J}$. Li, C. Guo, Y. Ma, Z. Wang, and J. Wang, Acta Mater. 90, 10-26 (2015).

${ }^{12}$ O. Krichevsky and J. Stevans, Phys. Rev. Lett. 70, 1473 (1993).

${ }^{13}$ T. Werz, M. Baumann, U. Wolfarm, and C. E. Krill, Mater. Charact. 90, 185 (2014).
${ }^{14} \mathrm{~J}$. D. Thompson, E. B. Gulsoy, and P. W. Voorhees, Acta Mater. 100, 282 (2015).

${ }^{15}$ D. R. H. Jones and G. J. May, Acta Metall. 23, 29-34 (1975).

${ }^{16}$ M. McLean, Scr. Metall. 9, 439-446 (1975).

17. P. Stark, Acta Metall. 26, 1133-1137 (1978).

18. P. Stark, Acta Metall. 26, 369-378 (1978).

${ }^{19}$ V. A. Snyder, N. Akaiwa, J. Alkemper, and P. W. Voorhees, Metall. Mater. Trans. A 30, 2341 (1999).

${ }^{20}$ A. Karma, Phys. Rev. Lett. 87, 115701 (2001).

${ }^{21}$ B. Echebarria, R. Folch, A. Karma, and M. Plapp, Phys. Rev. E 70, 061604 (2004).

${ }^{22}$ M. Ohno and K. Matsuura, Phys. Rev. E 79, 031603 (2009).

${ }^{23}$ M. Ohno, T. Takaki, and Y. Shibuta, Phys. Rev. E 96, 033311 (2017).

${ }^{24}$ T. Takaki, M. Ohno, T. Shimokawabe, and T. Aoki, Acta Mater. 81, 272-283 (2014).

${ }^{25}$ K. Glasner, J. Comput. Phys. 174, 695 (2001).

${ }^{26} \mathrm{M}$. Ohno, T. Takaki, and Y. Shibuta, Phys. Rev. E 93, 012802 (2016).

${ }^{27} \mathrm{M}$. Ohno, T. Takaki, and Y. Shibuta, J. Comput. Phys. 335, 621 (2017).

${ }^{28}$ A. J. Ardell, Metall. Trans. 3, 1395 (1972).

${ }^{29}$ J. A. Marqusee and J. Ross, J. Chem. Phys. 81, 976 (1984).

${ }^{30}$ N. Ofori-Opoku, V. Fallah, M. Greenwood, S. Esmaeili, and N. Provatas, Phys. Rev. B 87, 134105 (2013).

${ }^{31}$ N. Smith and N. Provatas, Phys. Rev. Mater. 1, 053407 (2017).

${ }^{32} \mathrm{M}$. Ohno, M. Yamashita, and K. Matsuura, Int. J. Heat Mass Transfer 132, 1004 (2019). 